\title{
Observations and answers to questions about edge-transitive maps*
}

\author{
Marston D.E. Conder ${ }^{\dagger}$ (D), Isabel Holm \\ Department of Mathematics, University of Auckland \\ Private Bag 92019, Auckland 1142, New Zealand \\ Thomas W. Tucker $\ddagger$ \\ Department of Mathematics, Colgate University, Hamilton, NY, USA
}

Received 21 February 2019, accepted 7 January 2020, published online 8 September 2020

\begin{abstract}
A map is a 2-cell embedding of a connected graph or multigraph on a closed surface, and a map is called edge-transitive if its automorphism group has a single orbit on edges. There are 14 classes of edge-transitive maps, determined by the effect of the automorphism group. In this paper we make some observations about these classes, and answer three open questions from a 2001 paper by Širán, Tucker and Watkins, by showing that (a) in each of the classes $1,2^{P}, 2^{P}$ ex $, 3,4^{P}$ and $5^{P}$, there exists a self-dual edge-transitive map, (b) there exists an edge-transitive map with simple underlying graph on an orientable surface of genus $g$ for every integer $g \geq 0$, and (c) there exists an orientable surface that carries an edge-transitive map of each of the 14 classes, and indeed that these three things still hold when we insist that both the map and its dual have simple underlying graph. We also give the maximum number of automorphisms of an edge-transitive map on an orientable surface of given genus $g>1$, and consider some special cases in which the automorphism group (or its subgroup of orientation-preserving automorphisms) is prescribed. For example, we show that a certain soluble group of order 576 is the smallest group that occurs as the automorphism group of some edge-transitive map in each of the 14 classes.
\end{abstract}

Keywords: Graph embedding, regular map, automorphism, edge-transitive, simple underlying graph, duality.

Math. Subj. Class. (2020): 57M15, 05C10, 05E18, 57M60

*The authors acknowledge helpful use of the MAGMA system [1] in constructing examples, searching for patterns in the resulting output, testing and confirming conjectures, and analysing specific examples.

${ }^{\dagger}$ Research supported in part by the N. Z. Marsden Fund, Grant UOA 1626

¥ The author has been supported by Simons Foundation Award 317689.

E-mail address: m.conder@auckland.ac.nz (Marston D.E. Conder), ihol325@aucklanduni.ac.nz (Isabel Holm), ttucker@colgate.edu (Thomas W. Tucker) 


\section{Introduction}

A regular map is a symmetric embedding of a connected graph or multigraph on a closed surface: the automorphism group of the embedding has a single orbit on 'flags' (which are like incident vertex-edge-face triples), or more loosely on 'arcs' (which are incident vertexedge pairs). The theory of such discrete objects has a long and interesting history, dating back to the work of Brahana, Burnside, Dyck and others, and recent work has produced infinite families of examples [10] as well as complete lists of those on hyperbolic surfaces of small genera $[3,5,6]$.

In contrast, relatively little is known about the more general case of edge-transitive maps, for which the automorphism group has a single orbit on edges. By work of Graver and Watkins [11], it is known that these can be divided into 14 classes according to certain properties (determined by automorphisms preserving a given edge or one of the vertices or faces incident with it). That work was taken further in [18] by Šrán, Tucker and Watkins, who showed there exist finite maps in each class. They also posed a number of questions, some of which were answered by Alen Orbanić in his $2006 \mathrm{PhD}$ thesis. Further questions were also posed by Orbanić et al in [17]. Some of the remaining questions and related ones were discussed at a BIRS workshop (on Symmetries of surfaces, maps and dessins) at Banff in September 2017, and in this paper we present the answers to many of those.

In particular, we answer Questions 3, 4 and 6 from [18], by showing the following:

(a) In each of the classes $1,2^{P}, 2^{P}$ ex $, 3,4^{P}$ and $5^{P}$, there exists a self-dual edgetransitive map, indeed one for which the map is non-degenerate, in that both the map and its dual have simple underlying graph;

(b) There exists a non-degenerate edge-transitive map on an orientable surface of genus $g$ for every integer $g \geq 0$, and

(c) There exists an orientable surface that carries a edge-transitive map of each of the 14 classes, and indeed a non-degenerate one of each class.

We also give the maximum number of automorphisms of an edge-transitive map on an orientable surface of given genus $g>1$, and consider some special cases in which the automorphism group (or its subgroup of orientation-preserving automorphisms) is prescribed. For example, we show that a certain soluble group of order 576 is the smallest group that occurs as the automorphism group of some edge-transitive map in each of the 14 classes.

Before doing that in Sections 3 to 8, we provide some further information about the 14 classes, including 'universal' groups that determine the effect of the automorphism group of every map in the class, and then we conclude the papers with some remarks and further questions.

Many of the findings we describe in this paper resulted from computations involving the universal groups and their quotients, using the MAGMA system, or were guided by them. In cases where the outcomes depended almost entirely on computations, we summarise them in tables in an Appendix at the end.

\section{Details and properties of the 14 classes}

In this section we present some background information on each class that can be helpful in constructing or analysing examples of edge-transitive maps. Much of this information can 
also be found in references [11] or [18] or [16] or [17], but unfortunately the table in [11] that was copied as Table I in [18] contains a number of shortcomings that make it difficult to follow. First, the columns headed $G_{v}$ and $G_{f}$ refer to the stabiliser of a particular vertex $v$ or face $f$, but for some classes the stabilisers of the vertex $u$ and/or face $g$ should be given as well. And in fact the column headed $G_{v}$ looks more appropriate for $G_{u}$, but even then, its entry for class $4^{*}$ should be $\left\langle\sigma_{u}^{4}, \theta_{u g}\right\rangle$, not $\left\langle\sigma_{u}^{4}, \theta_{u f}\right\rangle$. Also in Table II of [18], some relations are missing, namely $\tau^{2}=1$ for type 4 , and $\lambda^{2}=1$ for type $4^{*}$, and $\phi^{2}=1$ for type $4^{P}$. The information below is more accurate and comprehensive.

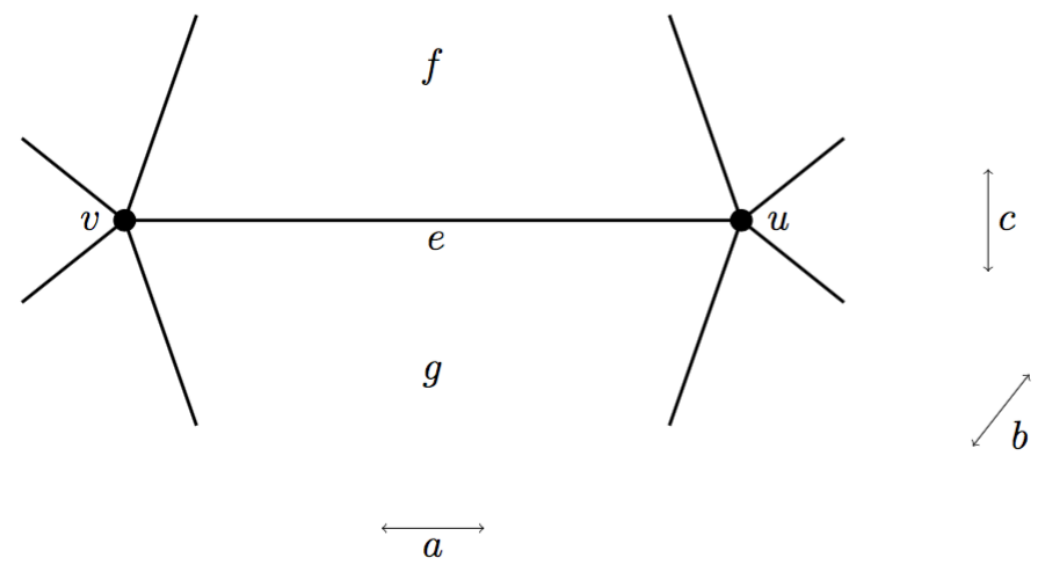

Figure 1: Reflecting generators for the universal group of class 1

In each case we let $e=\{u, v\}$ be a given edge, and let $f$ and $g$ be the faces incident with $e$, as illustrated in Figure 1.

Associated with each class is a universal group $U$, which has the property that if $M$ is any ET map of the given class, then its automorphism group $\operatorname{Aut}(M)$ is a quotient of $U$. This group $U$ is generated by particular elements that can be described in terms of their effect on the vertices, edges and faces labelled in Figure 1. Here we compose automorphisms from left to right.

Conversely, if $A$ is any quotient of $U$ in which the images of the generators and certain other elements have the appropriate orders (to avoid collapse), then there exists an ET map $M$ on which $A$ acts edge-transitively as a group of automorphisms, in the appropriate way. This map can be constructed using cosets of the stabilisers of the vertices $u$ and $v$, the edge $e$ and the faces $f$ and $g$ coming from Figure 1, in a similar way to the well-known construction for regular maps from groups (see [3], for example).

Class 1 consists of the fully regular maps. In this case, the universal group is

$$
U_{1}=\left\langle a, b, c \mid a^{2}=b^{2}=c^{2}=(a c)^{2}=1\right\rangle
$$

where $a, b$ and $c$ are automorphisms that act like local reflections, such that the stabilisers of the vertex $u$, edge $e$ and face $f$ are the subgroups generated by $\{b, c\},\{a, c\}$ and $\{a, b\}$ respectively. (These correspond to the elements $\lambda_{e}, \theta_{u f}$ and $\tau_{e}$ in the notation of [18].)

The universal group $U$ for any one of the 14 classes can be embedded into the group $U_{1}$ above, as a subgroup of index dividing 4, with transversal a subgroup of $\langle a, c\rangle$. We give this embedding, which is unique except for classes $4,4^{*}$ and $4^{P}$, where there are two possibilities (that can be interchanged under conjugation by $a, c$ and either $a$ or $c$, respectively). 
In each case, we also give generators for the orientation-preserving subgroup (which is the intersection of $U$ with the subgroup $\langle a b, b c\rangle$ of $U_{1}$ ) in the orientable case, and state whether or not the automorphism group of a map in the given class acts transitively on vertices, on faces, and/or on Petrie polygons of the map. Note that when the action (on vertices, faces, or Petrie polygons) is not transitive, there are two orbits (since each edge is incident with at most two vertices, and most two faces, and at most two Petrie polygons). Then we describe the stabilisers of the vertices $u$ and $v$, the edge $e$, and the faces $f$ and $g$, in terms of both generators for $U$ and generators for $U_{1}$. We also indicate the effect of an orientation-reversing element (usually but not always $b$ ) by conjugation on the generators for the orientation-preserving subgroup, when such an element exists, and similarly, the effect of a map duality on the chosen set of generators for $U$, in the orientable case.

Finally, for each element $g \in\{a, c, a c\}$ in the stated transversal for $U$ in $U_{1}$ (but lying outside $U$ ), we describe the effect of the automorphism $\psi_{g}$ of $U$ induced by conjugation by $g$, taking each generator $h$ of $U$ to $g^{-1} h g$. These $\psi_{g}$ are what we may call 'barred' automorphisms, in the sense that if $A$ is the automorphism group of an ET map in the given class, then $A$ has no automorphism that has the same effect on the images in $A$ of the generators of $U$ as $\psi_{g}$ has on the generators themselves.

\section{Class 1}

Universal group: $U=\left\langle x, y, z \mid x^{2}=y^{2}=z^{2}=(x z)^{2}=1\right\rangle$;

Embedding of generators in $U_{1}:(x, y, z) \mapsto(a, b, c)$, with transversal $\{1\}$;

Orientation-preserving subgroup $U^{+}$is generated by $r=x y=a b$ and $s=x z=a c$ (and $r^{-1} s=y z=b c$ ), subject to the single relation $s^{2}=1$;

Automorphism group of map is transitive on vertices, faces and Petrie polygons;

Stabiliser of vertex $u$ is generated by $\{y, z\}=\{b, c\}$;

Stabiliser of vertex $v$ is generated by $\{x y x, z\}=\{a b a, c\}$;

Stabiliser of edge $e$ is generated by $\{x, z\}=\{a, c\}$;

Stabiliser of face $f$ is generated by $\{x, y\}=\{a, b\}$;

Stabiliser of face $g$ is generated by $\{x, z y z\}=\{a, c b c\}$;

A reflection (by $a$ ) takes $(r, s)=(a b, a c) \mapsto(b a, c a)=\left(r^{-1}, s^{-1}\right)$;

An orientable duality takes $(a, b, c) \mapsto(c, b, a)$, preserving class 1 ;

There are no barred automorphisms.

\section{Class 2}

Universal group: $U=\left\langle x, y, z \mid x^{2}=y^{2}=z^{2}=1\right\rangle$;

Embedding of generators in $U_{1}:(x, y, z) \mapsto(b, c, a b a)$, with transversal $\{1, a\}$;

Orientation-preserving subgroup $U^{+}$is generated by $r=x y=b c$ and $s=z y=a b a c=a b c a$, and is free of rank 2;

Automorphism group of map is transitive on faces and Petrie polygons but not on vertices;

Stabiliser of vertex $u$ is generated by $\{x, y\}=\{b, c\}$;

Stabiliser of vertex $v$ is generated by $\{y, z\}=\{c, a b a\}$;

Stabiliser of edge $e$ is generated by $\{y\}=\{c\}$;

Stabiliser of face $f$ is generated by $\{x, z\}=\{b, a b a\}$;

Stabiliser of face $g$ is generated by $\{y x y, y z y\}=\{c b c, a c b c a\}=\{c b c, c a b a c\}$;

A reflection (by $c$ ) takes $(r, s)=(b c, a b a c) \mapsto(c b, c a b a)=\left(r^{-1}, s^{-1}\right)$;

An orientable map duality takes $(b, c, a b a) \mapsto(b, a, c b c)$, interchanging classes 2 and $2^{*}$; 
Extending automorphism $\psi_{a}$ takes $(x, y, z)=(b, c, a b a) \mapsto(a b a, c, b)=(z, y, x)$.

\section{Class $2^{*}$}

Universal group: $U=\left\langle x, y, z \mid x^{2}=y^{2}=z^{2}=1\right\rangle$;

Embedding of generators in $U_{1}:(x, y, z) \mapsto(a, b, c b c)$, with transversal $\{1, c\}$;

Orientation-preserving subgroup $U^{+}$is generated by $r=x y=a b$ and $s=y z=(b c)^{2}$, and is free of rank 2;

Automorphism group of map is transitive on vertices and Petrie polygons but not on faces;

Stabiliser of vertex $u$ is generated by $\{y, z\}=\{b, c b c\}$;

Stabiliser of vertex $v$ is generated by $\{x y x, x z x\}=\{a b a, a c b c a\}=\{a b a, c a b a c\}$;

Stabiliser of edge $e$ is generated by $\{x\}=\{a\}$;

Stabiliser of face $f$ is generated by $\{x, y\}=\{a, b\}$;

Stabiliser of face $g$ is generated by $\{x, z\}=\{a, c b c\}$;

A reflection (by $b$ ) takes $(r, s)=\left(a b,(b c)^{2}\right) \mapsto\left(b a,(c b)^{2}\right)=\left(r^{-1}, s^{-1}\right)$;

An orientable map duality takes $(a, b, c b c) \mapsto(c, b, a b a)$, interchanging classes $2^{*}$ and 2 ;

Extending automorphism $\psi_{c}$ takes $(x, y, z)=(a, b, c b c) \mapsto(a, c b c, b)=(x, z, y)$.

Class $2^{P}$

Universal group: $U=\left\langle x, y, z \mid x^{2}=y^{2}=z^{2}=1\right\rangle$;

Embedding of generators in $U_{1}:(x, y, z) \mapsto(a c, b, c b c)$, with transversal $\{1, a\}$ or $\{1, c\}$;

Orientation-preserving subgroup $U^{+}$is generated by $r=x=a c, s=y z=(b c)^{2}$ and $t=y x z=b a b c$, subject to the two relations $r^{2}=\left(s t^{-1}\right)^{2}=1$;

Automorphism group of map is transitive on vertices and faces but not on Petrie polygons;

Stabiliser of vertex $u$ is generated by $\{y, z\}=\{b, c b c\}$;

Stabiliser of vertex $v$ is generated by $\{x z x, x y x\}=\{a b a, a c b c a\}=\{a b a, c a b a c\}$;

Stabiliser of edge $e$ is generated by $\{x\}=\{a c\}$;

Stabiliser of face $f$ is generated by $\{y, x z x\}=\{b, a b a\}$;

Stabiliser of face $g$ is generated by $\{z, x y x\}=\{c b c, a c b c a\}=\{c b c, c a b a c\}$;

A reflection (by $b$ ) takes

$$
(r, s, t)=\left(a c,(b c)^{2}, b a b c\right) \mapsto\left(b a c b,(c b)^{2}, a b c b\right)=\left(t s^{-1}, s^{-1}, r s^{-1}\right) ;
$$

An orientable map duality takes $(a c, b, c b c) \mapsto(a c, b, a b a)$, preserving class $2^{P}$;

Extending automorphism $\psi_{c}$ takes $(x, y, z)=(a c, b, c b c) \mapsto(c a, c b c, b)=(x, z, y)$.

\section{Class 2ex}

Universal group: $U=\left\langle x, y \mid x^{2}=1\right\rangle$;

Embedding of generators in $U_{1}:(x, y) \mapsto(c, a b)$, with transversal $\{1, a\}$ or $\{1, b\}$;

Orientation-preserving subgroup $U^{+}$is generated by $r=y=a b$ and $s=x y x=c a b c$, and is free of rank 2;

Automorphism group of map is transitive on vertices, faces and Petrie polygons;

Stabiliser of vertex $u$ is generated by $\left\{x, y^{-1} x y\right\}=\{c, b c b\}$;

Stabiliser of vertex $v$ is generated by $\left\{x, y x y^{-1}\right\}=\{c, a b c b a\}=\{c,(a b a) c(a b a)\}$;

Stabiliser of edge $e$ is generated by $\{x\}=\{c\}$;

Stabiliser of face $f$ is generated by $\{y\}=\{a b\}$;

Stabiliser of face $g$ is generated by $\{x y x\}=\{c a b c\}=\{a c b c\}$;

A reflection (by $c$ ) takes $(r, s)=(a b, c a b c) \mapsto(c a b c, a b)=(s, r)$; 
An orientable map duality takes $(c, a b) \mapsto(a, c b)$, interchanging classes 2 ex and $2^{*}$ ex;

Extending automorphism $\psi_{a}$ takes $(x, y)=(c, a b) \mapsto(c, b a)=\left(x^{-1}, y^{-1}\right)$.

\section{Class $2^{*}$ ex}

Universal group: $U=\left\langle x, y \mid x^{2}=1\right\rangle$;

Embedding of generators in $U_{1}:(x, y) \mapsto(a, b c)$, with transversal $\{1, b\}$ or $\{1, c\}$;

Orientation-preserving subgroup $U^{+}$is generated by $r=y=b c$ and $s=x y x=a b c a$, and is free of rank 2;

Automorphism group of map is transitive on vertices, faces and Petrie polygons;

Stabiliser of vertex $u$ is generated by $\{y\}=\{b c\}$;

Stabiliser of vertex $v$ is generated by $\{x y x\}=\{a b c a\}=\{a b a c\}$;

Stabiliser of edge $e$ is generated by $\{x\}=\{a\}$;

Stabiliser of face $f$ is generated by $\left\{x, y x y^{-1}\right\}=\{a, b a b\}$;

Stabiliser of face $g$ is generated by $\left\{x, y^{-1} x y\right\}=\{a, c b a b c\}$;

A reflection (by $a$ ) takes $(r, s)=(b c, a b c a) \mapsto(a b c a, b c)=(s, r)$;

An orientable map duality takes $(a, b c) \mapsto(c, b a)$, interchanging classes $2^{*}$ ex and 2ex;

Extending automorphism $\psi_{c}$ takes $(x, y)=(a, b c) \mapsto(a, c b)=\left(x^{-1}, y^{-1}\right)$.

Class $2^{P}$ ex

Universal group: $U=\left\langle x, y \mid x^{2}=1\right\rangle$;

Embedding of generators in $U_{1}:(x, y) \mapsto(a c, a b)$, with transversal $\{1, a\}$;

Orientation-preserving subgroup $U^{+}$is equal to $U$;

Automorphism group of map is transitive on vertices, faces and Petrie polygons;

Stabiliser of vertex $u$ is generated by $\left\{y^{-1} x\right\}=\{b c\}$;

Stabiliser of vertex $v$ is generated by $\{y x\}=\{a b a c\}=\{a b c a\}$;

Stabiliser of edge $e$ is generated by $\{x\}=\{a c\}$;

Stabiliser of face $f$ is generated by $\{y\}=\{a b\}$;

Stabiliser of face $g$ is generated by $\{x y x\}=\{c b a c\}=\{c b c a\}$;

There is no orientation-reversing automorphism in $U$;

An orientable map duality takes $(a c, a b) \mapsto(a c, b c)$, preserving class $2^{P}$ ex;

Extending automorphism $\psi_{a}$ takes $(x, y)=(a c, a b) \mapsto(c a, b a)=\left(x^{-1}, y^{-1}\right)$.

\section{Class 3}

Universal group: $U=\left\langle x, y, z, w \mid x^{2}=y^{2}=z^{2}=w^{2}=1\right\rangle$;

Embedding of generators in $U_{1}:(x, y, z, w) \mapsto(b, c b c, a c b c a, a b a)$,

with transversal $\{1, a, c, a c\}$;

This group $U$ is also a subgroup of index 2 in the universal groups for classes $2,2^{*}$ and $2^{P}$ (but not in the universal groups for classes $2 \mathrm{ex}, 2^{*} \mathrm{ex}$ or $2^{P} \mathrm{ex}$ );

Orientation-preserving subgroup $U^{+}$is generated by $r=w x=(a b)^{2}$ and $s=x y=(b c)^{2}$ and $t=z x=(a c b)^{2}=(c a b)^{2}$, and is free of rank 3;

Automorphism group of map is not transitive on vertices, faces or Petrie polygons;

Stabiliser of vertex $u$ is generated by $\{x, y\}=\{b, c b c\}$;

Stabiliser of vertex $v$ is generated by $\{w, z\}=\{a b a, a c b c a\}=\{a b a, c a b a c\}$;

Stabiliser of edge $e$ is trivial;

Stabiliser of face $f$ is generated by $\{x, w\}=\{b, a b a\}$; 
Stabiliser of face $g$ is generated by $\{y, z\}=\{c b c, a c b c a\}$;

A reflection (by $b$ ) takes

$$
(r, s, t)=\left((a b)^{2},(b c)^{2},(a c b)^{2}\right) \mapsto\left((b a)^{2},(c b)^{2},(b a c)^{2}\right)=\left(r^{-1}, s^{-1}, t^{-1}\right) ;
$$

An orientable duality takes $(b, c b c, a c b c a, a b a) \mapsto(b, a b a, a c b c a, c b c)$ or $(a c b c a, c b c, b, a b a)$, preserving class 3 ;

Extending automorphisms $\psi_{a}, \psi_{c}$ and $\psi_{a c}$ respectively take $(x, y, z, w)=\left(b, b^{c}, b^{a c}, b^{a}\right)$ to $\left(b^{a}, b^{a c}, b^{c}, b\right),\left(b^{c}, b, b^{a}, b^{a c}\right)$ and $\left(b^{a c}, b^{a}, b, b^{c}\right)$, that is, to $(w, z, y, x),(y, x, w, z)$, and $(z, w, x, y)$.

\section{Class 4}

Universal group: $U=\left\langle x, y, z \mid x^{2}=y^{2}=1\right\rangle$

Embedding of generators in $U_{1}:(x, y, z) \mapsto(b, c b c, a c b a)$, with transversal $\{1, a, c, a c\}$;

This group $U$ is also a subgroup of index 2 in the universal group for class 2

(but not in the universal groups for classes $2^{*}, 2^{P}, 2 \mathrm{ex}, 2^{*} \mathrm{ex}$ or $2^{P} \mathrm{ex}$ );

Orientation-preserving subgroup $U^{+}$is generated by $r=y x=(c b)^{2}$ and $s=z=a c b a$ and $t=y z x=c b a b a b$, and is free of rank 3;

Automorphism group of map is transitive on faces and Petrie polygons but not on vertices;

Stabiliser of vertex $u$ is generated by $\{x, y\}=\{b, c b c\}$;

Stabiliser of vertex $v$ is generated by $\{z\}=\{a c b a\}=\{c a b a\}$;

Stabiliser of edge $e$ is trivial;

Stabiliser of face $f$ is generated by $\left\{x, z^{-1} y z\right\}=\{b, a b a b a b a\}$;

Stabiliser of face $g$ is generated by

$\left\{y, z x z^{-1}\right\}=\{c b c, a c b a b a b c a\}=\{c b c, a(c b c) a(c b c) a(c b c) a\}$;

A reflection (by $b$ ) takes

$(r, s, t)=\left((c b)^{2}, a c b a, c b a b a b\right) \mapsto\left((b c)^{2}, b a c b a b, b c b a b a\right)=\left(r^{-1}, r^{-1} t, r^{-1} s\right) ;$

An orientable duality takes $(b, c b c, a c b a) \mapsto(b, a b a, c a b c)$, interchanging classes 4 and $4^{*}$; Extending automorphism $\psi_{c}$ takes $(x, y, z)=(b, c b c, a c b a) \mapsto(c b c, b, a b a c)=\left(y, x, z^{-1}\right)$.

\section{Class $4^{*}$}

Universal group: $U=\left\langle x, y, z \mid x^{2}=y^{2}=1\right\rangle$;

Embedding of generators in $U_{1}:(x, y, z) \mapsto(b, a b a, c a b c)$, with transversal $\{1, a, c, a c\}$;

This group $U$ is also a subgroup of index 2 in the universal group for class $2^{*}$

(but not in the universal groups for classes $2,2^{P}, 2 \mathrm{ex}, 2^{*}$ ex or $2^{P} \mathrm{ex}$ );

Orientation-preserving subgroup $U^{+}$is generated by $r=y x=(a b)^{2}$ and $s=z=c a b c$ and $t=y z x=a b c b c b$, and is free of rank 3;

Automorphism group of map is transitive on vertices and Petrie polygons but not on faces;

Stabiliser of vertex $u$ is generated by $\left\{x, z^{-1} y z\right\}=\{b, c b c b c b c\}$;

Stabiliser of vertex $v$ is generated by

$\left\{y, z x z^{-1}\right\}=\{a b a, c a b c b c b a c\}=\{a b a, c(a b a) c(a b a) c(a b a) c\}$;

Stabiliser of edge $e$ is trivial;

Stabiliser of face $f$ is generated by $\{x, y\}=\{b, a b a\}$;

Stabiliser of face $g$ is generated by $\{z\}=\{c a b c\}=\{a c b c\}$;

A reflection (by $b$ ) takes

$(r, s, t)=\left((a b)^{2}, c a b c, a b c b c b\right) \mapsto\left((b a)^{2}, b a c b c b, b a b c b c\right)=\left(r^{-1}, r^{-1} t, r^{-1} s\right) ;$

An orientable duality takes $(b, a b a, c a b c) \mapsto(b, c b c, a c b a)$, interchanging classes $4^{*}$ and 4 ; 
Extending automorphism $\psi_{a}$ takes $(x, y, z)=(b, a b a, c a b c) \mapsto(a b a, b, c b c a)=\left(y, x, z^{-1}\right)$.

\section{Class $4^{P}$}

Universal group: $U=\left\langle x, y, z \mid x^{2}=y^{2}=1\right\rangle$;

Embedding of generators in $U_{1}:(x, y, z) \mapsto(b, a c b c a, a b c)$, with transversal $\{1, a, c, a c\}$;

This group $U$ is also a subgroup of index 2 in the universal group for class $2^{P}$

(but not in the universal groups for classes $2,2^{*}, 2 \mathrm{ex}, 2^{*}$ ex or $2^{P}$ ex);

Orientation-preserving subgroup $U^{+}$is generated by

$r=z x=a b c b$ and $s=x z=b a b c$ and $t=z y=a b a b a c$, and is free of rank 3;

Automorphism group of map is transitive on vertices and faces but not on Petrie polygons;

Stabiliser of vertex $u$ is generated by $\left\{x, z^{-1} y z\right\}=\{b, c b c b c b c\}$;

Stabiliser of vertex $v$ is generated by $\left\{y, z x z^{-1}\right\}=\{a c b c a, a b c b c b a\}=\{c a b a c$, $(a b a) c(a b a) c(a b a)\}$;

Stabiliser of edge $e$ is trivial;

Stabiliser of face $f$ is generated by $\left\{x, z y z^{-1}\right\}=\{b, a b a b a b a\}$;

Stabiliser of face $g$ is generated by $\left\{y, z^{-1} x z\right\}=\{a c b c a, c b a b a b c\}$;

A reflection (by $b$ ) takes

$(r, s, t)=(a b c b, b a b c, a b a b a c) \mapsto(b a b c, a b c b, b a b a b a c b)=\left(s, r, s t^{-1} r\right) ;$

An orientable duality takes $(b, a c b c a, a b c) \mapsto(b, a c b c a, c b a)$ or $(a c b c a, b, a b c)$,

preserving class $4^{P}$;

Extending automorphism $\psi_{a c}$ takes

$(x, y, z)=(b, a c b c a, a b c) \mapsto(a c b c a, b, c b a)=\left(y, x, z^{-1}\right)$.

\section{Class 5}

Universal group: $U=\langle x, y \mid-\rangle$ (free of rank 2);

Embedding of generators in $U_{1}:(x, y) \mapsto(b c, a b c a)$, with transversal $\{1, a, c, a c\}$;

This group $U$ is also a subgroup of index 2 in the universal groups for classes $2,2^{*}$ ex and $2^{P}$ ex (but not in the universal groups for classes $2^{*}, 2^{P}$ or $2 \mathrm{ex}$ );

Orientation-preserving subgroup $U^{+}$is equal to $U$;

Automorphism group of map is transitive on faces and Petrie polygons but not on vertices;

Stabiliser of vertex $u$ is generated by $\{x\}=\{b c\}$;

Stabiliser of vertex $v$ is generated by $\{y\}=\{a b c a\}=\{a b a c\}$;

Stabiliser of edge $e$ is trivial;

Stabiliser of face $f$ is generated by $\left\{y x^{-1}\right\}=\left\{(a b)^{2}\right\}$;

Stabiliser of face $g$ is generated by $\left\{y^{-1} x\right\}=\left\{c(a b)^{2} c\right\}=\{a(c b c) a(c b c)\}$;

There is no orientation-reversing automorphism in $U$;

An orientable duality takes $(b c, a b c a) \mapsto(b a, c b a c)$, interchanging classes 5 and $5^{*}$;

Extending automorphisms $\psi_{a}, \psi_{c}$ and $\psi_{a c}$ respectively take $(x, y)=(b c, a b c a)$ to $(a b c a, b c)=(y, x),(c b, c a b a)=\left(x^{-1}, y^{-1}\right)$ and $(a c b a, c b)=\left(y^{-1}, x^{-1}\right)$.

\section{Class $5^{*}$}

Universal group: $U=\langle x, y \mid-\rangle$ (free of rank 2);

Embedding of generators in $U_{1}:(x, y) \mapsto(a b, c a b c)$, with transversal $\{1, a, c, a c\}$;

This group $U$ is also a subgroup of index 2 in the universal groups for classes $2^{*}, 2 \mathrm{ex}$ and $2^{P}$ ex (but not in the universal groups for classes $2,2^{P}$ or $2^{*} \mathrm{ex}$ ); 
Orientation-preserving subgroup $U^{+}$is equal to $U$;

Automorphism group of map is transitive on vertices and Petrie polygons but not on faces;

Stabiliser of vertex $u$ is generated by $\left\{x^{-1} y\right\}=\left\{(b c)^{2}\right\}$;

Stabiliser of vertex $v$ is generated by $\left\{x y^{-1}\right\}=\left\{a(b c)^{2} a\right\}=\{(a b a) c(a b a) c\}$;

Stabiliser of edge $e$ is trivial;

Stabiliser of face $f$ is generated by $\{x\}=\{a b\}$;

Stabiliser of face $g$ is generated by $\{y\}=\{c a b c\}=\{a c b c\}$;

There is no orientation-reversing automorphism in $U$;

An orientable duality takes $(a b, c a b c) \mapsto(c b, a c b a)$, interchanging classes $5^{*}$ and 5 ;

Extending automorphisms $\psi_{a}, \psi_{c}$ and $\psi_{a c}$ respectively take $(x, y)=(a b, c a b c)$ to $(b a, c b c a)=\left(x^{-1}, y^{-1}\right),(c a b c, a b)=(y, x)$ and $(c b a c, b a)=\left(y^{-1}, x^{-1}\right)$.

\section{Class $5^{P}$}

Universal group: $U=\langle x, y \mid-\rangle$ (free of rank 2);

Embedding of generators in $U_{1}:(x, y) \mapsto(a b c, a c b)$, with transversal $\{1, a, c, a c\}$;

This group $U$ is also a subgroup of index 2 in the universal groups for classes $2^{P}$, 2ex and $2^{*}$ ex (but not in the universal groups for classes $2,2^{*}$ or $2^{P}$ ex);

Orientation-preserving subgroup $U^{+}$is generated by $r=x y=(a b)^{2}$ and $s=y^{-1} x=(b c)^{2}$ and $t=x y^{-1}=a(b c)^{2} a$, and is free of rank 3;

Automorphism group of map is transitive on vertices and faces but not on Petrie polygons; Stabiliser of vertex $u$ is generated by $\left\{y^{-1} x\right\}=\left\{(b c)^{2}\right\}$;

Stabiliser of vertex $v$ is generated by $\left\{x y^{-1}\right\}=\left\{a(b c)^{2} a\right\}=\{(a b a) c(a b a) c\}$;

Stabiliser of edge $e$ is trivial;

Stabiliser of face $f$ is generated by $\{x y\}=\left\{(a b)^{2}\right\}$;

Stabiliser of face $g$ is generated by $\{y x\}=\left\{c(a b)^{2} c\right\}=\{a(c b c) a(c b c)\}$;

Conjugation by the orientation-reversing automorphism $x=a b c$ takes

$(r, s, t)=\left((a b)^{2},(b c)^{2}, a(b c)^{2} a\right) \mapsto(c a b a b c, c b a b c b c a b c, b c b c)=\left(t^{-1} r s, s^{-1} r^{-1} t r s, s\right) ;$

An orientable duality takes $(a b c, a c b) \mapsto(c b a, a c b)$ or $(a b c, b c a)$, preserving class $5^{P}$;

Extending automorphisms $\psi_{a}, \psi_{c}$ and $\psi_{a c}$ respectively take $(x, y)=(a b c, a c b)$ to $(b c a, c b a)=\left(y^{-1}, x^{-1}\right),(c a b, a b c)=(y, x)$ and $(c b a, b a c)=\left(x^{-1}, y^{-1}\right)$.

It can also be helpful to see how the 14 universal subgroups can be embedded not just in $U_{1}$ but also in each other. These inclusions are illustrated in Figure 2.

Indeed here we may note that the 14 classes correspond precisely to the 14 conjugacy classes of subgroups of $U_{1}$ that are complementary to some subgroup of the edge-stabiliser $\langle a, c\rangle$, and that this gives a purely algebraic way of finding them, much more easily than in the approach taken in [11]. It is also easy to determine these classes using the combinatorial approach of 'symmetry type graphs' in [17].

\section{Maximum orders of automorphism groups for ET maps of genus greater than 1}

Theorem 3.1. Let $A$ be a group of automorphisms of an edge-transitive map on some orientable surface of genus $g>1$, or some non-orientable surface of genus $p>2$. Then $|A| \leq|A|_{\text {maxo }}$ or $|A| \leq|A|_{\text {maxnono, }}$ respectively, where $|A|_{\text {maxo }}$ and $|A|_{\text {maxnono }}$ are given in Table 1 for each of the 14 classes of edge-transitive maps. Moreover, these bounds are 


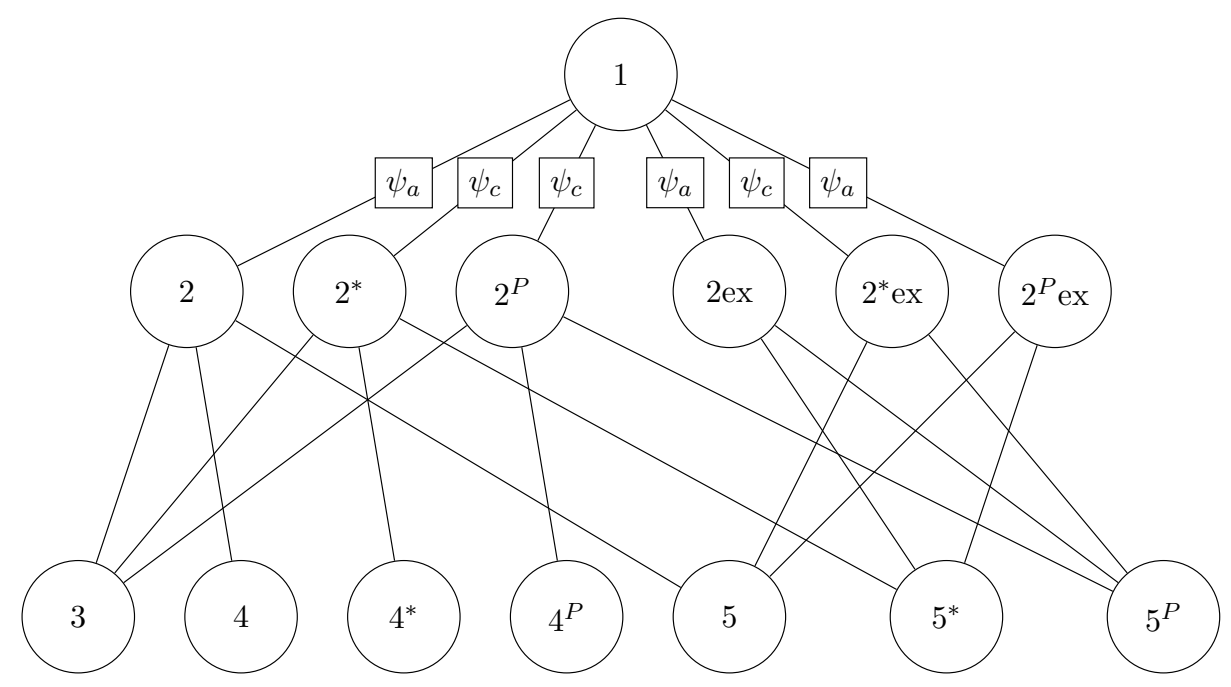

Figure 2: Inclusions among the universal groups of the 14 classes

sharp for certain values of $g$ and $p$ in each class.

Proof. First, we might as well take $A$ as the full automorphism group of the map in each case, and then the bounds can be proved easily using the orders of stabilisers of vertices, edges and faces, and the Euler-Poincaré formula.

For example, in class 1 we know that if $k=o(x y)=o(a b)$ and $m=o(y z)=o(b c)$, then $|V|=\left|A: A_{u}\right|=|A| / k$ and $|E|=\left|A: A_{e}\right|=|A| / 4$ and $|F|=\left|A: A_{f}\right|=|A| / m$, so the Euler characteristic is $\chi=|V|-|E|+|F|=|A|(1 / k-1 / 4+1 / m)$. Then the maximum possible negative value of this is $-|A| / 84$, achievable when $\{k, m\}=\{3,7\}$, and giving $|A| \leq-84 \chi=168(g-1)$ or $84(p-2)$ in the orientable and non-orientable cases, respectively. Similarly, for class 4 , we have $|V|=\left|A: A_{u}\right|+\left|A: A_{v}\right|=|A| / o(z)+$ $|A| /(2 o(x y))$ and $|E|=|A|$ and $|F|=\left|A: A_{f}\right|=|A| / o\left(x z y z^{-1}\right)$, and this gives the maximum possible negative value of $\chi=|V|-|E|+|F|$ as $-|A| / 24$, achievable when $\left(o(z), o(x y), o\left(x z y z^{-1}\right)\right)=(3,4,1)$ or $(3,1,4)$. The other cases are similar, and are left as an exercise for the reader.

Sharpness can be proved by exhibiting examples for which the bounds are attained.

If $M$ is a fully regular map of type $\{3,7\}$ or $\{7,3\}$, such as Klein's map of genus 3 , then $\operatorname{Aut}(M)$ is generated by elements $a, b, c$ satisfying $a^{2}=b^{2}=c^{2}=(a b)^{3}=(b c)^{7}=$ $(a c)^{2}=1$, and $|\operatorname{Aut}(M)|=168(g-1)$ or $84(p-2)$, and this proves sharpness for class 1 . But then also the triple $(x, y, z)=(a, b, c)$ satisfies the defining relations for the universal group of class 2 maps, but there exists no automorphism of the group that takes $(x, y, z)$ to $(z, y, x)$, since $o(x y)=3$ while $o(z y)=o(y z)=7$. Hence this triple gives an ET map in class 2, with $\chi=(|A| / 6+|A| / 14)-|A| / 2+|A| / 4=-|A| / 84$, and therefore the same genus and the same number of automorphisms as $M$. Also its dual is in class $2^{*}$ and has the same genus and same automorphism group as well.

Similarly, if $M$ is an orientably-regular but chiral map of type $\{3,7\}$ or $\{7,3\}$, then it has class $2^{P}$ ex and $84(g-1)$ automorphisms. Also any irreflexible generating pair 


\begin{tabular}{|l|c|c|l|}
\hline \hline Classes & $|A|_{\text {maxo }}$ & $|A|_{\text {maxnono }}$ & Sufficient conditions for achieving maximum \\
\hline \hline 1 & $168(g-1)$ & $84(p-2)$ & $(o(x y), o(y z))=(3,7)$ or $(7,3)$ \\
\hline $2,2^{*}$ & $168(g-1)$ & $84(p-2)$ & $\{o(x y), o(y z), o(x z)\}=\{2,3,7\}$ \\
\hline $2^{P}$ & $24(g-1)$ & $12(p-2)$ & $(o(y z), o(x y x z))=(2,3)$ or $(3,2)$ \\
\hline $2 \mathrm{ex}, 2^{*} \mathrm{ex}$ & $48(g-1)$ & $24(p-2)$ & $\left(o(y), o\left(x y^{-1} x y\right)\right)=(3,4)$ \\
\hline $2^{P} \mathrm{ex}$ & $84(g-1)$ & N/A & $\{o(y), o(x y)\}=\{3,7\}$ \\
\hline 3 & $24(g-1)$ & $12(p-2)$ & $\{o(x y), o(z w), o(y z), o(x w)\}=\{2,2,2,3\}$ \\
\hline $4,4^{*}$ & $48(g-1)$ & $24(p-2)$ & $\left(o(z), o(x y), o\left(x z y z^{-1}\right)\right)$ \\
& & & $(3,4,1)$ or $(3,1,4)$ \\
\hline $4^{P}$ & $8(g-1)$ & $4(p-2)$ & $\left(o\left(x z^{-1} y z\right), o\left(x z y z^{-1}\right)\right)=(1,2)$ or $(2,1)$ \\
\hline $5,5^{*}$ & $84(g-1)$ & N/A & $\left\{o(x), o(y), o\left(x y^{-1}\right)\right\}=\{2,3,7\}$ \\
\hline $5^{P}$ & $12(g-1)$ & $6(p-2)$ & $\left\{o(x y), o\left(x y^{-1)}\right\}=\{2,3\}\right.$ \\
\hline \hline
\end{tabular}

Table 1: Bounds on the number of automorphisms

$(x, y)$ for $\operatorname{Aut}(M)$ such that $x^{3}=y^{7}=\left(x^{-1} y\right)^{2}=1$ satisfies the conditions for a canonical generating pair for the automorphism group of a map of class 5 or $5^{*}$, with $\chi=(|A| / 3+|A| / 7)-|A|+|A| / 2=-|A| / 42$, and hence the same genus and same number of automorphisms as $M$.

Small examples of maps in classes $2^{P}, 3$ and $4^{P}$ attaining the upper bound on the number automorphisms can be found in Sections 4.5 of Alen Orbanić's thesis [16]. In particular, his lists include orientable maps with $(g,|A|)=(6,60),(2,24)$ and $(15,112)$, respectively, and non-orientable maps with $(p,|A|)=(30,168),(3,12)$ and $(16,56)$, respectively.

Additional computations (using MAGMA [1]) give examples attaining the bounds for the remaining five classes. In class $2 \mathrm{ex}$, there exists an orientable map of genus $g=26$ with $|A|=1200=48(g-1)$, and a non-orientable map of genus $p=1346$ with $|A|=32256=$ $24(p-2)$, and their duals give corresponding examples in class $2^{*}$ ex. Also the same thing happens in classes 4 and $4^{*}$. Finally, in class $5^{P}$ there exists an orientable map of genus $g=14$ with $|A|=156=12(g-1)$, as given also in $[16, \S 4.5]$, and a non-orientable map of genus $p=226$ with $|A|=1344=6(p-2)$. This completes the proof.

Many other examples than those mentioned in the last two paragraphs of the above proof can be found. Further details are available form the first author upon request.

\section{Self-dual non-degenerate orientable ET maps}

In this brief section, we answer Question 6 of [18], about self-duality for ET maps in classes $1,2^{P}, 2^{P}$ ex $, 3,4^{P}$ and $5^{P}$. In fact we can do even more, by proving the following:

Theorem 4.1. In each of the classes $1,2^{P}, 2^{P}$ ex, $3,4^{P}$ and $5^{P}$, there exist self-dual edgetransitive orientable maps such that the map and its dual have simple underlying graph.

Proof. Computations using MAGMA produce the non-degenerate self-dual maps summarised in Table 3, with defining relations for their automorphism groups given in Table 4, in the Appendix. 


\section{Edge-transitive maps with simple underlying graphs}

Question 3 in [18] asked the following: Does every closed orientable surface support some non-degenerate, edge-transitive map? (It is not difficult to construct degenerate regular maps (of class 1) on orientable surfaces of all possible genera.)

Examples of small genera are already widely known, such as the five Platonic maps on the sphere (genus 0 ) and the regular maps $\{4,4\}_{2 q}$ on the torus (see [10]).

We can complete an answer to the above question positively by proving the following:

Theorem 5.1. For every integer $g \geq 2$, there exists an edge-transitive map of class 2 on the orientable surface of genus $g$, such that both the map and its dual have simple underlying graph (indeed with underlying graph being a complete bipartite graph in each case).

We have two proofs of this theorem. We give one of them, and then give a brief description of the other, based on an alternative construction for the family of maps involved.

Proof. We start by taking the following group, which is a quotient of the universal group for ET maps of class 2 (obtained by adding two extra relations):

$$
G=\left\langle x, y, z \mid x^{2}=y^{2}=z^{2}=[x, z]=(x y z y)^{2}=1\right\rangle .
$$

In this group $G$, the subgroup $N$ generated by $a=(x y)^{2}=[x, y]$ and $b=(y z)^{2}=[y, z]$ is normal, with

$$
\begin{gathered}
a^{x}=a^{-1}, a^{y}=a^{-1} \text { and } a^{z}=z x y x y z=z x(y x y z)^{-1}=z x z y x y=(x y)^{2}=a, \\
\text { and } b^{x}=x y z y z x=(x y z y)^{-1} z x=y z y x z x=(y z)^{2}=b, b^{y}=b^{-1} \text { and } b^{z}=b^{-1} \text {, }
\end{gathered}
$$

and the quotient $G / N$ is elementary abelian of order 8 . Moreover, by ReidemeisterSchreier theory (explained in $[12, \S 12 \& \S 13]$ and implemented as the Rewrite command in MAGMA [1]), the subgroup $N$ is free abelian of rank 2 .

Now for any even positive integers $k$ and $m$, let $N^{(k, m)}$ be the subgroup of $N$ generated by $a^{k / 2}=(x y)^{k}$ and $b^{m / 2}=(y z)^{m}$. Then $N^{(k, m)}$ is normal in $G$, with conjugation of its generators by $x, t=y$ and $z$ following the same pattern as given for $a$ and $b$ above, and the quotient $Q^{(k, m)}=G / N^{(k, m)}$ is isomorphic to an extension of $N / N^{(k, m)} \cong C_{k / 2} \times C_{m / 2}$ by $G / N \cong C_{2} \times C_{2} \times C_{2}$. In particular, $Q^{(k, m)}=G / N^{(k, m)}$ has order $2 k m$.

We now use these quotients $Q^{(k, m)}$ of $G$ to construct bipartite ET maps of class 2 with the required properties, one for each choice of the pair $(k, m)$ with $k \neq m$. For notational convenience, from now on we will let $x, y, z, a$ and $b$ denote the images in $Q^{(k, m)}$ of the elements given above. Equivalently, we simply assume that the elements $a=(x y)^{2}$ and $b=(y z)^{2}$ have orders $k / 2$ and $m / 2$ respectively. Also we now define $Q=Q^{(k, m)}$, and let $N$ be the normal subgroup generated by $a$ and $b$ in $Q$, isomorphic to $C_{k / 2} \times C_{m / 2}$, with quotient $Q / N \cong C_{2} \times C_{2} \times C_{2}$.

For the underlying graph of the map for a given pair $(k, m)$, we take the vertices of one part as the $m$ right cosets of the dihedral subgroup $V_{1}$ of order $2 k$ generated by $x$ and $y$, and the vertices of the other part as the $k$ right cosets of the dihedral subgroup $V_{2}$ of order $2 m$ generated by $y$ and $z$, and define adjacency by non-trivial intersection.

For example, the vertex $V_{1}=\langle x, y\rangle$ is adjacent to the $k$ cosets of $V_{2}=\langle y, z\rangle$ of the form $V_{2}(x y)^{i}$ for $i \in \mathbb{Z}_{k}$, with $V_{2}(x y)^{i} x=V_{2}(y x)^{-i} x=V_{2}(x y)^{-i-1}$ for all such $i$. Note that the coset intersections are given by $V_{1} \cap V_{2}(x y)^{i}=\left\{(x y)^{i}, y(x y)^{i}\right\}=$ $\left\{(x y)^{i},(x y)^{-i-1} x\right\}$ for $i \in \mathbb{Z}_{k}$. In particular, the valency of the vertex $V_{1}$ is $k$, and hence 
$V_{1}$ is adjacent to every vertex of the second part. Similarly, the vertex $V_{2}=\langle y, z\rangle$ has neighbours $V_{1}(z y)^{j}$ for $0 \leq j<m$, and hence it is adjacent to every one of the $m$ vertices of the first part.

For the faces of the map, we use the right cosets of the subgroup $H$ of order 4 generated by $x$ and $z$, and define incidence again by non-empty intersection. (We preserve the symbol $F$ for the set of faces.) For example, since $H=\{1, x, z, x z\}$, the face $H$ itself is incident with the vertices $V_{1}$ and $V_{1} z$ in the first part, and the vertices $V_{2}$ and $V_{2} x$ in the second part. Here we note that $z \notin V_{1}=\langle x, y\rangle$, for otherwise $Q=\langle x, y, z\rangle=\langle x, y\rangle$ which has order $2 k<2 k m$, and similarly $x \notin V_{2}=\langle y, z\rangle$, because the latter has order $2 m<2 k m$. In particular, the face $H$ has four different vertices, and hence also four different edges.

Also the group $Q$ acts by right multiplication on this map, with two orbits of sizes $m$ and $k$ on vertices, namely the two parts of the graph, and a single orbit on faces, and a single orbit on edges. Indeed the edges can be identified with right cosets of the subgroup $K$ of order 2 generated by $y$, with $K(x y)^{i}=\left\{(x y)^{i}, y(x y)^{i}\right\}=\left\{(x y)^{i},(x y)^{-i-1} x\right\}$ for all $i \in \mathbb{Z}_{k}$. It follows that every vertex of the first part has valency $k$, and every vertex of the second part has valency $m$, and hence the graph is isomorphic to the complete bipartite graph $K_{m, k}$. Similarly, the map has $2 \mathrm{~km} / 4=\mathrm{km} / 2$ faces, all of which have length 4 (with four distinct vertices), and so the dual of the map is simple too.

Next, if $k \neq m$ then the parts of the underlying graph have different sizes, so the map cannot lie in class 1 , and hence it has class 2. (This also follows from the fact that $Q$ has no automorphism taking $(x, y, z)$ to $(z, y, x)$, since the orders of $x y$ and $z y$ are $k$ and $m$.)

Finally, the map is orientable, since the subgroup generated by $x y$ and $z y\left(=(y z)^{-1}\right)$ has index 2 in $Q$ (with image $C_{2} \times C_{2}$ in $Q / N \cong C_{2} \times C_{2} \times C_{2}$ ), and its Euler characteristic is $\chi=|V|-|E|+|F|=(m+k)-m k+(m k / 2)=m+k-m k / 2$, so its genus $g$ is $(2-\chi) / 2=(4-2 m-2 k+m k) / 4=(k-2)(m-2) / 4$. Taking $m=4$ gives genus $g=(k-2) / 2=k / 2-1$, which can be any integer greater than 1 when $k / 2>2$.

This completes the construction and proof.

An alternative way of constructing these maps is to add six extra relations to the universal group for ET maps of class 3, to give the group with presentation

$$
\begin{aligned}
\langle x, y, z, w| x^{2} & =y^{2}=z^{2}=w^{2}=[x, z]= \\
& \left.=[y, z]=[x, w]=[y, w]=(x y)^{k}=(z w)^{m}=1\right\rangle,
\end{aligned}
$$

which is isomorphic to the direct product of $\langle x, y\rangle \cong D_{k}$ and $\langle z, w\rangle \cong D_{m}$.

The above map with underlying graph $K_{m, k}$ can be constructed from this group, but the group admits an automorphism of order 2 that takes $(x, y, z, w)$ to $(y, x, w, z)$, and another taking $(x, y, z, w)$ to $(z, w, x, y)$ when $k=m$, and hence the map has class 1 or class 2 , depending on whether or not $k=m$. The proof is straightforward.

Next, a natural question related to Question 3 in [18] (but not posed in [18]) is the following: Does there exist a simple graph $X$ that is the underlying graph of a map in each of the 14 classes of edge-transitive maps?

Note that any such graph must be arc-transitive and hence regular (because it underlies an ET map of class 1), with valency divisible by 4 (because it underlies an ET map of class 5), and also bipartite (because it underlies an ET map of class 2). Accordingly, there are natural candidates to check. In an early search we found that the complete bipartite graph $K_{8,8}$ underlies ET maps of 11 of the 14 classes, namely all of them except classes $2^{*}$ ex, $2^{P}$ ex and 5. A little further work led us to a positive answer to the question: 
Theorem 5.2. In each of the 14 classes of edge-transitive maps, there exists an orientable map with underlying graph isomorphic to the complete bipartite graph $K_{16,16}$.

Proof. Computations using MAGMA produce the maps with underlying graph $K_{16,16}$ summarised in Table 5, with defining relations for their automorphism groups given in Table 6, in the Appendix.

\section{Orientable ET maps of genus 14}

Question 4 in [18, Section 6] is the following: What is the largest number of automorphismgroup types for edge-transitive maps contained by one surface? Is there some surface that supports all 14 types? We can now answer both parts of this.

Theorem 6.1. The orientable surface of genus 14 carries edge-transitive maps of all 14 classes.

Proof. Computations using MAGMA produce the maps summarised in Table 7, with defining relations for their automorphism groups given in Table 8, in the Appendix.

In particular, the answer to the second part of Question 4 in [18, Section 6] is "Yes", and the answer to the first part is 14 . Also we can prove that 14 is the smallest genus for which this happens, by MAGMA computations and Theorem 3.1 (to bound the order of the groups required for consideration in a search for examples). Again, further details are available from the first author upon request. On the other hand, there are other surfaces of higher genus that carry ET maps of all 14 classes, as explained in the next section.

\section{Non-degenerate orientable ET maps of genus 17}

A natural extension of Question 4 in [18, Section 6] is the following: Is there some surface that supports at least one non-degenerate ET map of each of the 14 classes? The answer to this question is also "Yes":

Theorem 7.1. The orientable surface of genus 17 carries edge-transitive maps of all 14 classes, with the property that the map and its dual have simple underlying graph.

Proof. Computations using MAGMA produce the non-degenerate maps summarised in Table 9, with defining relations for their automorphism groups given in Table 10, in the Appendix.

\section{Edge-transitive maps with prescribed automorphism group(s)}

Another very natural question is this: Which finite groups occur as the automorphism group of at least one ET map in each of the 14 classes? This same question can be asked with a restriction to simple or non-degenerate maps.

Clearly a necessary condition is that the group can be generated by two elements. On the other hand, for orientable ET maps with more than two edges, it cannot be cyclic, because in [18] it was shown that if an orientable ET map $M$ has at least three edges, then $\operatorname{Aut}(M)$ is a non-abelian group, except in the case where $M$ has class $4^{P}$, and $\operatorname{Aut}(M)$ is isomorphic to the direct product $C_{n} \times C_{2}$ where $n \equiv 2 \bmod 4$. In that exceptional case the stabiliser of every vertex and every face is the subgroup of order 4 generated by 
the images of the two involutory generators $x$ and $y$ of the universal group for class $4^{P}$, and the underlying graph of the map has double edges. Similarly it can be shown easily that if a non-orientable ET map $M$ has at least three edges and $\operatorname{Aut}(M)$ is abelian, then either $M$ has class 3 (with two vertices and eight edges) and $\operatorname{Aut}(M) \cong C_{2} \times C_{2} \times C_{2}$, or $M$ has class 4 or $4^{*}$ and $\operatorname{Aut}(M) \cong C_{n} \times C_{2}$ for some even $n$, or $M$ has class $4^{P}$ and $\operatorname{Aut}(M) \cong C_{n} \times C_{2}$ for some $n$ divisible by 4 . Again in these cases the underlying graph of the map has multiple edges.

We can also extend these observations to abelian groups that occur as the orientationpreserving subgroup of $\operatorname{Aut}(M)$ for some orientable ET map $M$. It is not difficult to show that there are no such maps in classes $2^{P}, 2 \mathrm{ex}, 2^{*} \mathrm{ex}, 2^{P}$ ex $, 4,4^{*}, 5,5^{*}$ and $5^{P}$ (mainly because the abelian groups involved admit outer automorphisms that put the map into a higher class), but there exist infinite families of examples in the other five classes, namely $1,2,2^{*}, 3$ and $4^{P}$.

In class 1 these maps have just one or two vertices, and just one or two faces, with multiple edges. In class $4^{P}$ the maps have $(|V|,|E|,|F|)=(k, 2 k n, k)$ for arbitrary integers $k \geq 1$ and $n \geq 2$ or 3 , but again none of them is simple. In class 2 , some of the maps are simple but all have non-simple dual, while in class $2^{*}$, all of the maps are nonsimple (but some have simple dual). The only class containing non-degenerate orientable ET maps is class 3 , and in these case there are four infinite families of examples with $(|V|,|E|,|F|)=(4 k+4,16 k, 4 k+4)$ and $\operatorname{Aut}(M) \cong C_{2 k} \times C_{2} \times C_{2}$ for all $k \geq 2$, with two infinite families containing self-dual examples, and the other two consisting of duals of each other.

Details are available from the first author upon request.

A major contribution in the non-abelian case for the above question was made by Gareth Jones in [13], where he considered this for non-abelian simple groups, the symmetric groups $S_{n}$, soluble groups, and nilpotent groups. In particular, he showed in [13, Theorem 1.2] that a given non-abelian simple group is the automorphism group of some ET map of a given class unless it appears in a list of known exceptions, copied in Table 2 below.

\begin{tabular}{|l|l|}
\hline \hline Class & Non-abelian simple groups that do not occur \\
\hline \hline 1 & $L_{3}(q), U_{3}(q), L_{4}\left(2^{e}\right), U_{4}(3), U_{5}(2), A_{6}, A_{7}, M_{11}, M_{22}, M_{23}, M c L$ \\
\hline $2,2^{*}, 2^{P}$ & $U_{3}(3)$ \\
\hline $2 \mathrm{ex}, 2^{*} \mathrm{ex}, 2^{P}$ ex & $L_{2}(q), L_{3}(q), U_{3}(q), A_{7}$ \\
\hline 3 & - \\
\hline $4,4^{*}, 4^{P}$ & - \\
\hline $5,5^{*}, 5^{P}$ & $L_{2}(q)$ \\
\hline \hline
\end{tabular}

Table 2: Non-occurrences of non-abelian simple groups for a given class of ET map

Note that in 11 of the 14 classes (all except $2^{P}$ ex, 5 and $5^{*}$ ), the maps are nonorientable, for the obvious reason that a non-abelian simple group has no subgroup of index 2 .

It follows easily from Table 2 that the smallest simple group that occurs for all 14 classes is the Suzuki group $S z(8)$, of order 29120. In fact, $S z(8)$ is the automorphism 
group of some non-degenerate ET map in every one of the 14 classes; details are available from the first author on request. The same holds for the next smallest examples, which are $M_{12}, J_{1}$ and $A_{9}$, and in particular, $A_{5}, A_{6}, A_{7}$ and $A_{8}$ do not occur in this way.

Indeed by [13, Theorem 1.1], the alternating group $A_{n}$ of degree $n$ is the automorphism group of some ET map in any given class, except in the following cases: $n \in\{3,4,6,7,8\}$ for class $1 ; n \in\{1,2,3,4\}$ for classes $2,2^{*}, 2^{P}$ and $3 ; n \in\{1,2,3,4,5,6,7\}$ for classes 2ex, $2^{*}$ ex and $2^{P}$ ex; $n \in\{1,2,3\}$ for classes $4,4^{*}$ and $4^{P}$; and $n \in\{1,2,3,4,5,6\}$ for classes $5,5^{*}$ and $5^{P}$. In particular, $A_{9}$ is the smallest alternating group that occurs for all 14 classes. Moreover, non-degenerate ET maps occur in all classes for $A_{9}$ (and $A_{10}$ ); details are available from the first author on request.

Similarly, by [13, Theorem 1.1], the symmetric group $S_{n}$ of degree $n$ is the automorphism group of some ET map in any given class, except in the following cases: $n=1$ for classes $2,2^{*}, 2^{P}, 3,4,4^{*}$ and $4^{P}$; and $n \in\{1,2,3,4,5\}$ for classes $2 \mathrm{ex}, 2^{*} \mathrm{ex}, 2^{P}$ ex, 5 , $5^{*}$ and $5^{P}$. In particular, $S_{6}$ is the smallest symmetric group that occurs for all 14 classes. Moreover, non-degenerate ET maps occur in all classes for $S_{6}$ (and $S_{7}, S_{8}, S_{9}$ and $S_{10}$ ); details are available from the first author on request.

Incidentally, the symmetric group $S_{6}$ is the smallest insoluble group that occurs as the automorphism group of some ET map in every one of the 14 classes, and again, the same holds under the assumption that the map is simple or non-degenerate.

Finally, we have the following:

Theorem 8.1. The smallest finite group $G$ that occurs as the automorphism group of some ET map in every one of the 14 classes is the 8654th group of order 576 (in the database of all groups of order up to 2000).

Proof. Computations using MAGMA produce maps of each class for this group, indeed non-degenerate maps of all 14 classes, and these are summarised in Table 11, with defining relations for their automorphism groups given in Table 12, in the Appendix. The same computations show that no group of smaller or equal order (necessarily divisible by 4) has the required property.

Note that just one of the maps given in Table 11 is non-orientable, namely the one in class $2^{P}$. There are also orientable examples in class $2^{P}$ with simple underlying graph, but none with simple dual. Again, further details about these maps are available from the first author on request.

\section{Some final remarks and questions}

Answers always raise more questions. We ask a few of them for this topic below.

For each of the 14 classes of ET maps, define the non-degenerate genus spectrum for that class to be the set of genera of orientable surfaces that carry a non-degenerate map of that class. By our Theorem 5.1 (and knowledge of ET maps of genus 0 and 1), the non-degenerate genus spectrum for class 2 (and therefore also $2^{*}$ ) is the set of all nonnegative integers. The corresponding question for classes 1 (regular maps) and/or $2^{P}$ ex (chiral maps), however, is a challenging open question (see [7, 8]).

Indeed, questions about genus spectra questions are notoriously difficult. Another example comes from group actions. Given a finite group $G$, its symmetric genus $\sigma(G)$ is defined as the smallest non-negative integer $g$ for which $G$ has a faithful action on some 
compact Riemann surface of genus $g$. Much but not all of the spectrum for $\sigma$ is known; see [9]. In contrast, the strong symmetric genus $\sigma^{o}(G)$ is the smallest $g$ such that $G$ has a faithful action on such a surface of genus $g$, preserving orientation, and the spectrum for $\sigma^{o}$ contains all non-negative integers, by a theorem of May and Zimmerman in [15], which incidentally uses the groups $C_{k} \times D_{m}$ in a similar way to the way we used the groups $D_{k} \times D_{m}$ in proving Theorem 5.1. The following question(s) could constitute a long-term project:

Question 9.1. What are the genus spectra for the 14 classes of ET maps, under the restriction to non-degenerate maps, or to simple maps, or to ET maps in general?

The symmetric groups $S_{n}$ play a prominent role in [18], producing ET maps in each class, but with restrictions on the congruence class $n$ modulo 12 . Those restrictions are removed in [13], where it is also shown that $S_{n}$ realises all classes for every $n \geq 6$, and $A_{n}$ realises all classes for every $n \geq 9$.

Similarly, the first author of this paper showed in [4] that $S_{n}$ and $A_{n}$ realise the upper

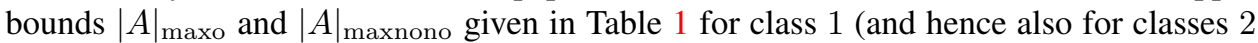
and $2^{*}$ ), for all but finitely many $n$, and many years later the same in [2, Theorem 6.3] for $A_{n}$ for class $2^{P}$ ex (and hence also classes 5 and $5^{*}$ ), again for all but finitely many $n$.

Question 9.2. For which other classes do $A_{n}$ and/or $S_{n}$ realise the upper bounds $|A|_{\text {maxo }}$ and $|A|_{\text {maxnono }}$ in Table 1, possibly with restrictions on $n$ ?

Edge-transitive maps on non-orientable surfaces have been largely ignored in the literature. Even in this paper, most of our theorems, examples and tables concern maps on orientable surfaces, and yet there should be non-orientable versions:

Question 9.3. What are the analogues of Theorems 4.1, 5.1, 5.2, 6.1 and 7.1 for nonorientable ET maps (in the 11 classes other than $2^{P}$ ex, 5 and $\left.5^{*}\right)$ ? How might the information given in the tables in the Appendix differ for non-orientable maps?

Finally, for a map on a non-orientable surface $S$ with automorphism group $G$, a standard technique is to pass to the orientable double cover of $S$, where $G \times C_{2}$ acts, with $G$ preserving orientation and $C_{2}$ orientation-reversing and fixed-point free (in effect, an antipodal symmetry). If the given map (on $S$ ) is edge-transitive, then so is the lifted map under the group $G \times C_{2}$. On the other hand, the lifted map may actually be 'unstable', in that it has a symmetry group larger than $G \times C_{2}$, which means that the lifted map may be in a different class, with larger edge-stabiliser. Many examples of this phenomenon have been provided recently by Gareth Jones [14].

Question 9.4. How common is instability for edge-transitive maps on non-orientable surfaces?

\section{ORCID iDs}

Marston D.E. Conder (D) https://orcid.org/0000-0002-0256-6978

Isabel Holm (D) https://orcid.org/0000-0002-6806-7514

Thomas W. Tucker (D) https://orcid.org/0000-0002-7868-6925 


\section{References}

[1] W. Bosma, J. Cannon and C. Playoust, The Magma algebra system. I. The user language, volume 24, pp. 235-265, 1997, doi:10.1006/jsco.1996.0125, computational algebra and number theory (London, 1993).

[2] E. Bujalance, M. D. E. Conder and A. F. Costa, Pseudo-real Riemann surfaces and chiral regular maps, Trans. Amer. Math. Soc. 362 (2010), 3365-3376, doi:10.1090/S0002-9947-10-05102-0.

[3] M. Conder and P. Dobcsányi, Determination of all regular maps of small genus, J. Combin. Theory Ser. B 81 (2001), 224-242, doi:10.1006/jctb.2000.2008.

[4] M. D. E. Conder, Generators for alternating and symmetric groups, J. London Math. Soc. (2) 22 (1980), 75-86, doi:10.1112/jlms/s2-22.1.75.

[5] M. D. E. Conder, Regular maps and hypermaps of Euler characteristic -1 to -200 , J. Combin. Theory Ser. B 99 (2009), 455-459, doi:10.1016/j.jctb.2008.09.003.

[6] M. D. E. Conder, Lists of regular and chiral maps on surfaces of Euler characteristic -1 to -600, 2012, https: / / www . math. auckland.ac.nz/ conder.

[7] M. D. E. Conder and J. Ma, Regular maps with simple underlying graphs, J. Combin. Theory Ser. B 110 (2015), 1-18, doi:10.1016/j.jctb.2014.07.001.

[8] M. D. E. Conder, J. Širáň and T. W. Tucker, The genera, reflexibility and simplicity of regular maps, J. Eur. Math. Soc. (JEMS) 12 (2010), 343-364, doi:10.4171/JEMS/200.

[9] M. D. E. Conder and T. W. Tucker, The symmetric genus spectrum of finite groups, Ars Math. Contemp. 4 (2011), 271-289, doi:10.26493/1855-3974.127.eb9.

[10] H. S. M. Coxeter and W. O. J. Moser, Generators and relations for discrete groups, volume 14 of Ergebnisse der Mathematik und ihrer Grenzgebiete [Results in Mathematics and Related Areas], Springer-Verlag, Berlin-New York, 4th edition, 1980.

[11] J. E. Graver and M. E. Watkins, Locally finite, planar, edge-transitive graphs, Mem. Amer. Math. Soc. 126 (1997), vi+75, doi:10.1090/memo/0601.

[12] D. L. Johnson, Topics in the theory of group presentations, volume 42 of London Mathematical Society Lecture Note Series, Cambridge University Press, Cambridge-New York, 1980.

[13] G. A. Jones, Automorphism groups of edge-transitive maps, Acta Math. Univ. Comenian. (N.S.) 88 (2019), 841-847.

[14] G. A. Jones, Unstable maps, Acta Math. Univ. Comenian. (N.S.) 88 (2019), 341-350, doi: 10.1007/s10623-019-00685-y.

[15] C. L. May and J. Zimmerman, There is a group of every strong symmetric genus, Bull. London Math. Soc. 35 (2003), 433-439, doi:10.1112/S0024609303001954.

[16] A. Orbanić, Edge-transitive maps, Ph.D. thesis, University of Ljubljana, 2006.

[17] A. Orbanić, D. Pellicer, T. Pisanski and T. W. Tucker, Edge-transitive maps of low genus, Ars Math. Contemp. 4 (2011), 385-402, doi:10.26493/1855-3974.249.3a6.

[18] J. Širáň, T. W. Tucker and M. E. Watkins, Realizing finite edge-transitive orientable maps, J. Graph Theory 37 (2001), 1-34, doi:10.1002/jgt.1000.abs. 


\section{Appendix}

Below are the tables mentioned in the proofs of Theorems 5.1, 5.2, 6.1, 7.1 and 4.1. Note that there are two tables for each theorem: the first gives details about a map from each of the relevant classes, and the second gives defining relations for the automorphism groups of those maps (in terms of the canonical generators for the relevant universal group).

The following notation is used in the odd-numbered tables. First 'SD' and 'NSD' indicate that the map is self-dual or non-self-dual, respectively. (We do not indicate 'NSD' in the obvious cases, where $|V| \neq|F|$.) Next, $C_{n}, D_{n}, A_{n}$ and $S_{n}$ denote the cyclic group of order $n$, the dihedral group of degree $n$ (and order $2 n$ ), and the alternating and symmetric groups of degree $n$, while $\operatorname{Group}(n, k)$ denotes the $k$ th group in the database of all groups of order up to 2000 (except 1024), available in the MAGMA system [1]. Finally, $V_{4}$ denotes the direct product $C_{2} \times C_{2}$ (the Sylow 2-subgroup of $A_{4}$ ), while $K \rtimes H$ denotes a semidirect product with kernel $K$ and complement $H$, and $C_{n} \rtimes_{k} C_{m}$ denotes the semi-direct product $\left\langle a, b \mid a^{m}=b^{n}=1, a^{-1} b a=b^{k}\right\rangle$.

\begin{tabular}{|c|c|c|c|c|c|c|c|l|}
\hline \hline Class & $|V|$ & $|E|$ & $|F|$ & Genus & $|A|$ & $A_{u}, A_{v}$ & $A_{f}, A_{g}$ & Comments \\
\hline \hline 1 & 4 & 6 & 4 & 0 & 24 & $D_{3}$ & $D_{3}$ & $\operatorname{Aut}(M) \cong S_{4}$ \\
\hline $2^{P}$ & 8 & 16 & 8 & 1 & 32 & $V_{4}$ & $V_{4}$ & $\begin{array}{l}\operatorname{Aut}(M) \cong \\
\operatorname{Group}(32,43)\end{array}$ \\
\hline $2^{P}$ ex & 5 & 10 & 5 & 1 & 20 & $C_{4}$ & $C_{4}$ & $\operatorname{Aut}(M) \cong C_{5} \rtimes_{2} C_{4}$ \\
\hline 3 & 12 & 32 & 12 & 5 & 32 & $D_{4}, V_{4}$ & $D_{4}, V_{4}$ & $\operatorname{Aut}(M) \cong D_{4} \times V_{4}$ \\
\hline $4^{P}$ & 12 & 48 & 12 & 13 & 48 & $V_{4}$ & $V_{4}$ & $\operatorname{Aut}(M) \cong A_{4} \times V_{4}$ \\
\hline $5^{P}$ & 14 & 42 & 14 & 8 & 42 & $C_{3}$ & $C_{3}$ & $\operatorname{Aut}(M) \cong C_{7} \rtimes_{2} C_{6}$ \\
\hline \hline
\end{tabular}

Table 3: Non-degenerate self-dual maps with the smallest number of edges in six of the 14 classes

\begin{tabular}{|c|l|}
\hline \hline Class & Defining relations for $A=\operatorname{Aut}(M)$ \\
\hline \hline 1 & $x^{2}=y^{2}=z^{2}=(x z)^{2}=(x y)^{3}=(y z)^{3}=1$ \\
\hline $2^{P}$ & $x^{2}=y^{2}=z^{2}=(y z)^{2}=(x z)^{4}=(x y x z)^{2}=(x y)^{3} z x z y=1$ \\
\hline $2^{P}$ ex & $x^{2}=y^{4}=x y x y^{2} x y^{-1}=1$ \\
\hline 3 & $\begin{array}{l}x^{2}=y^{2}=z^{2}=w^{2}=(x z)^{2}=(y z)^{2}=(y w)^{2}=(z w)^{2}= \\
(x y)^{4}=x y w x w y=1\end{array}$ \\
\hline $4^{P}$ & $x^{2}=y^{2}=(x y)^{2}=(x z)^{3}=[x, z]^{2}=[x y, z]=1$ \\
\hline $5^{P}$ & $x^{6}=x y^{2} x^{2} y^{-1}=x y^{-2} x^{2} y=1$ \\
\hline \hline
\end{tabular}

Table 4: Defining relations for the automorphism groups of the maps in Table 3 


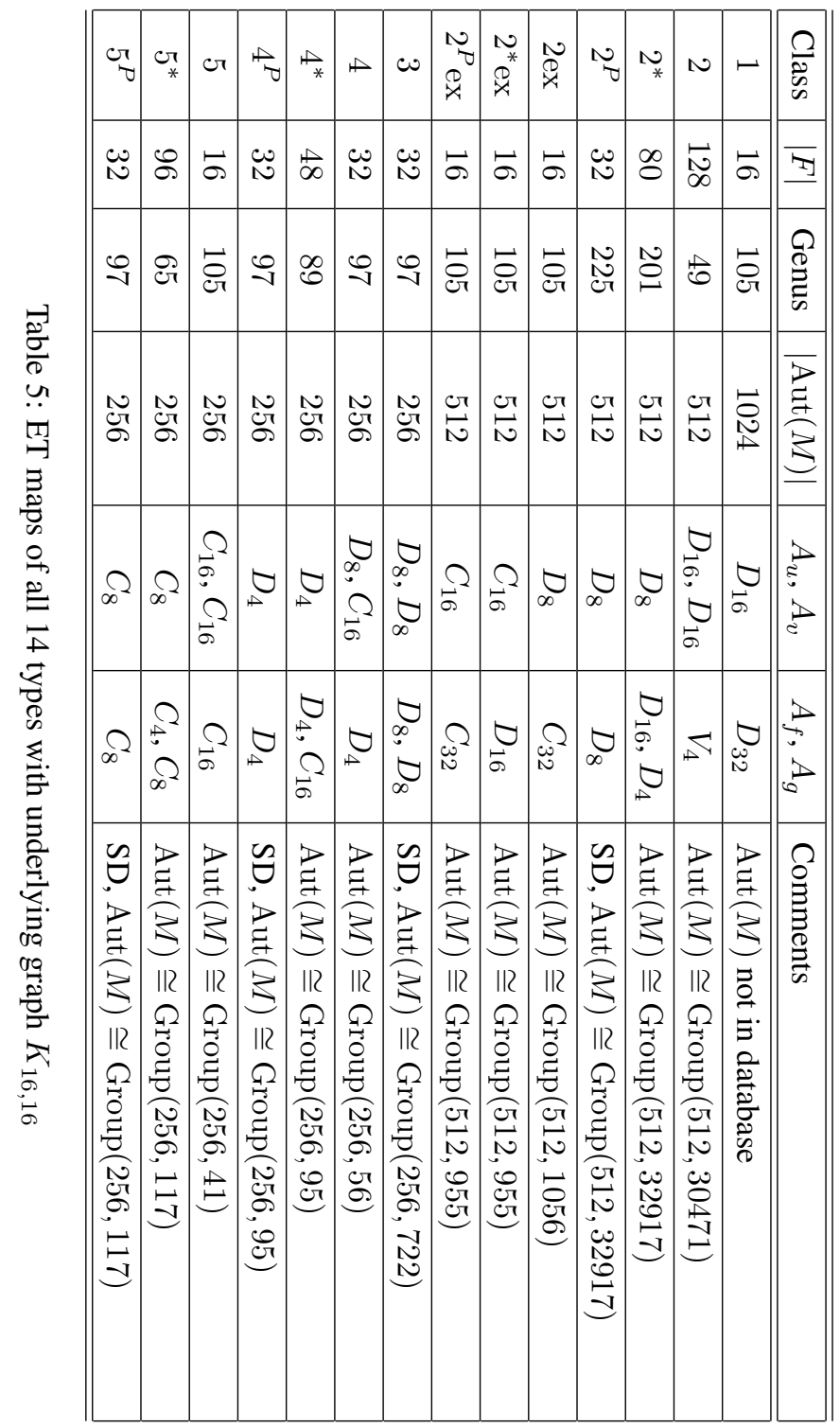




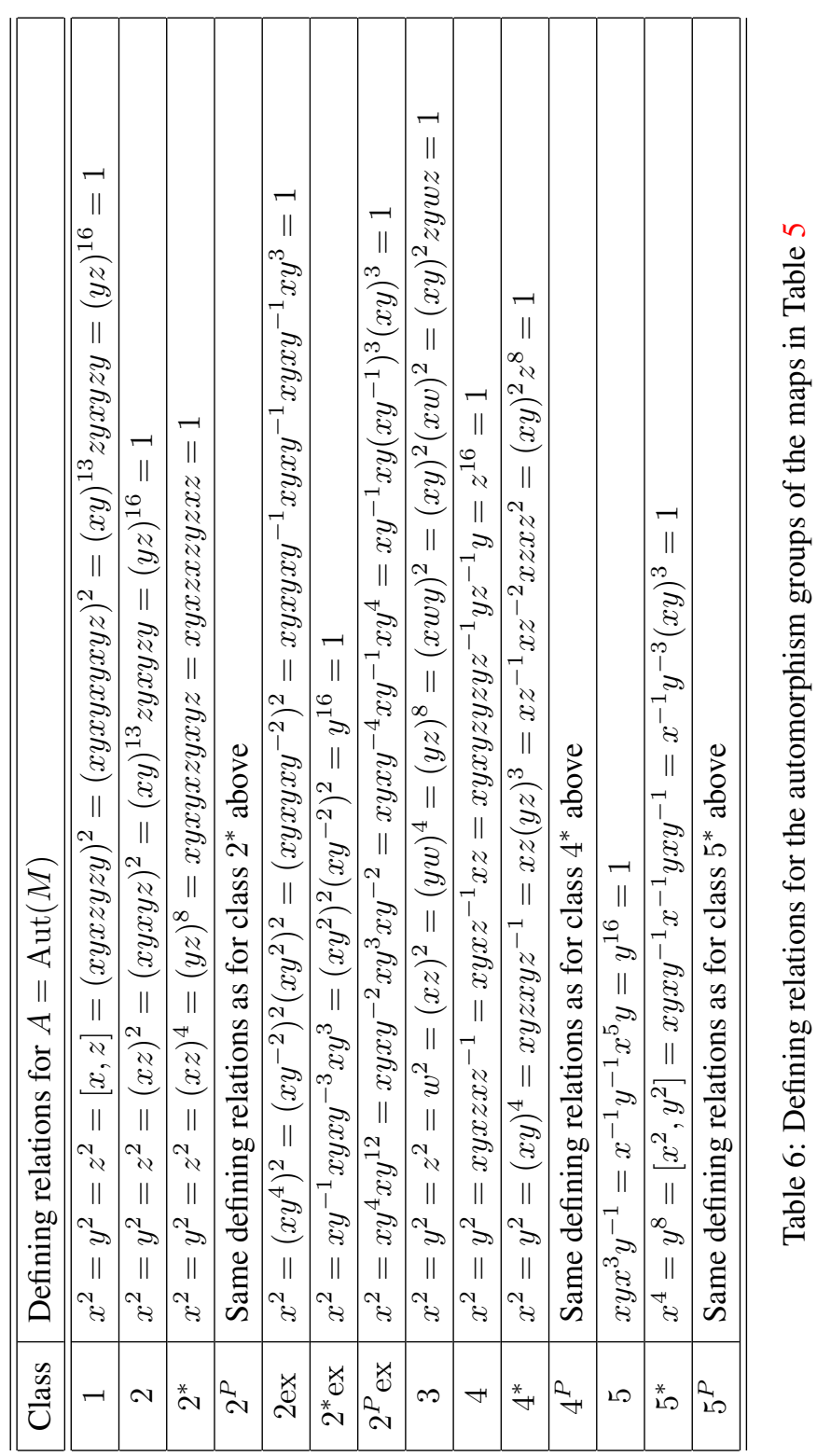




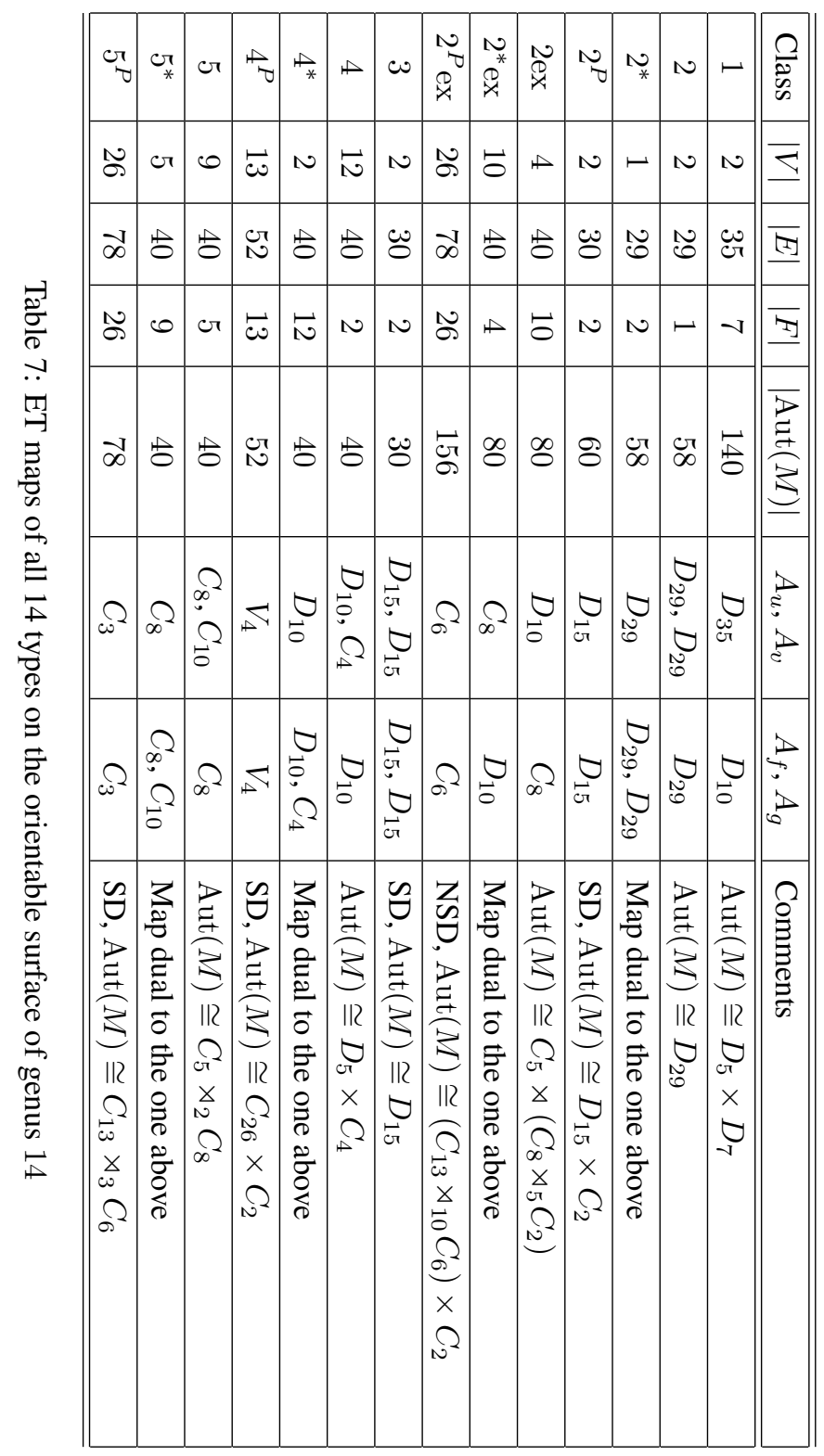




\begin{tabular}{|c|l|}
\hline \hline Class & Defining relations for $A=\operatorname{Aut}(M)$ \\
\hline \hline 1 & $x^{2}=y^{2}=z^{2}=(x z)^{2}=(x y x y z)^{2}=(x y z)^{2}(y z)^{5}=1$ \\
\hline 2 & $x^{2}=y^{2}=z^{2}=x z y z=(x y)^{14} x z=1$ \\
\hline $2^{*}$ & $x^{2}=y^{2}=z^{2}=y z x z=(y x)^{14} y z=1$ \\
\hline $2^{P}$ & $x^{2}=y^{2}=z^{2}=(x y z)^{2}=(x z)^{2}(y z)^{3}=(x y)^{6}=1$ \\
\hline $2 \mathrm{ex}$ & $x^{2}=y^{8}=\left[x, y^{4}\right]=(x y)^{4} y^{4}=x y x y^{2} x y^{2} x y^{-1}=1$ \\
\hline $2^{*} \mathrm{ex}$ & Same defining relations as for class $2 \mathrm{ex}$ above \\
\hline $2^{P}$ ex & $x^{2}=y^{6}=\left(x y x y^{2}\right)^{2}=(x y)^{3}\left(x y^{-1}\right)^{3}=x y^{-2} x y^{2} x y^{3} x y^{3}=1$ \\
\hline 3 & $x^{2}=y^{2}=z^{2}=w^{2}=x y x z w y=x y x w x=(x z)^{3}=1$ \\
\hline 4 & $x^{2}=y^{2}=z^{4}=[x, z]=[x z, y]=(x y)^{3}(z y)^{2}=1$ \\
\hline $4^{*}$ & Same defining relations as for class 4 above \\
\hline $4^{P}$ & $x^{2}=y^{2}=(x y)^{2}=[x, z]=[y, z]=y z^{13}=1$ \\
\hline 5 & $x^{2} y x^{-2} y=x y^{3} x^{-1} y^{-1}=x y^{-1} x^{3} y^{2}=1$ \\
\hline $5^{*}$ & Same defining relations as for class 5 above \\
\hline $5^{P}$ & $x^{6}=(x y)^{3}=\left(x y^{-1}\right)^{3}=x y^{-1} x^{-1} y^{3}=1$ \\
\hline \hline
\end{tabular}

Table 8: Defining relations for the automorphism groups of the maps in Table 7 


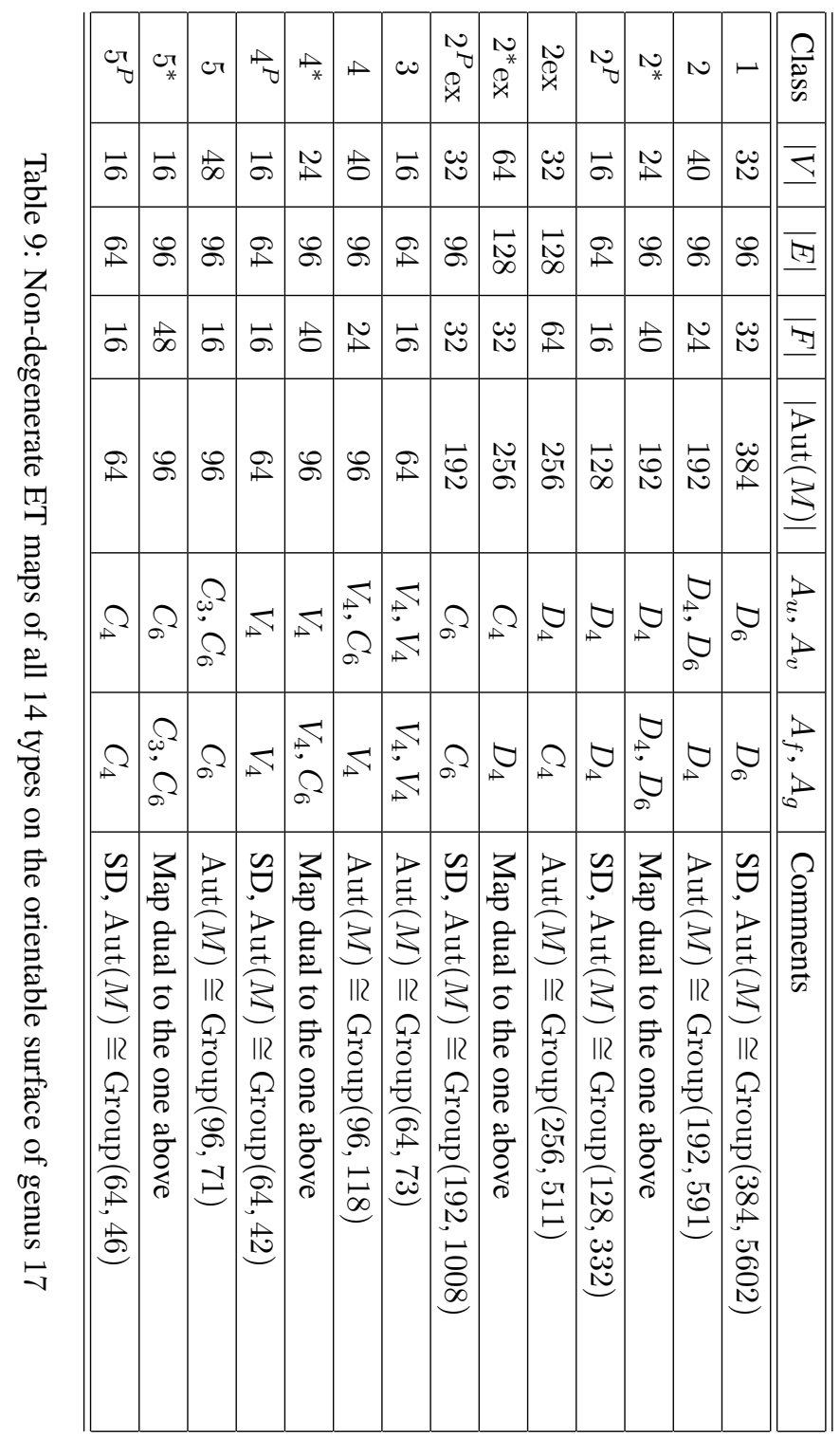




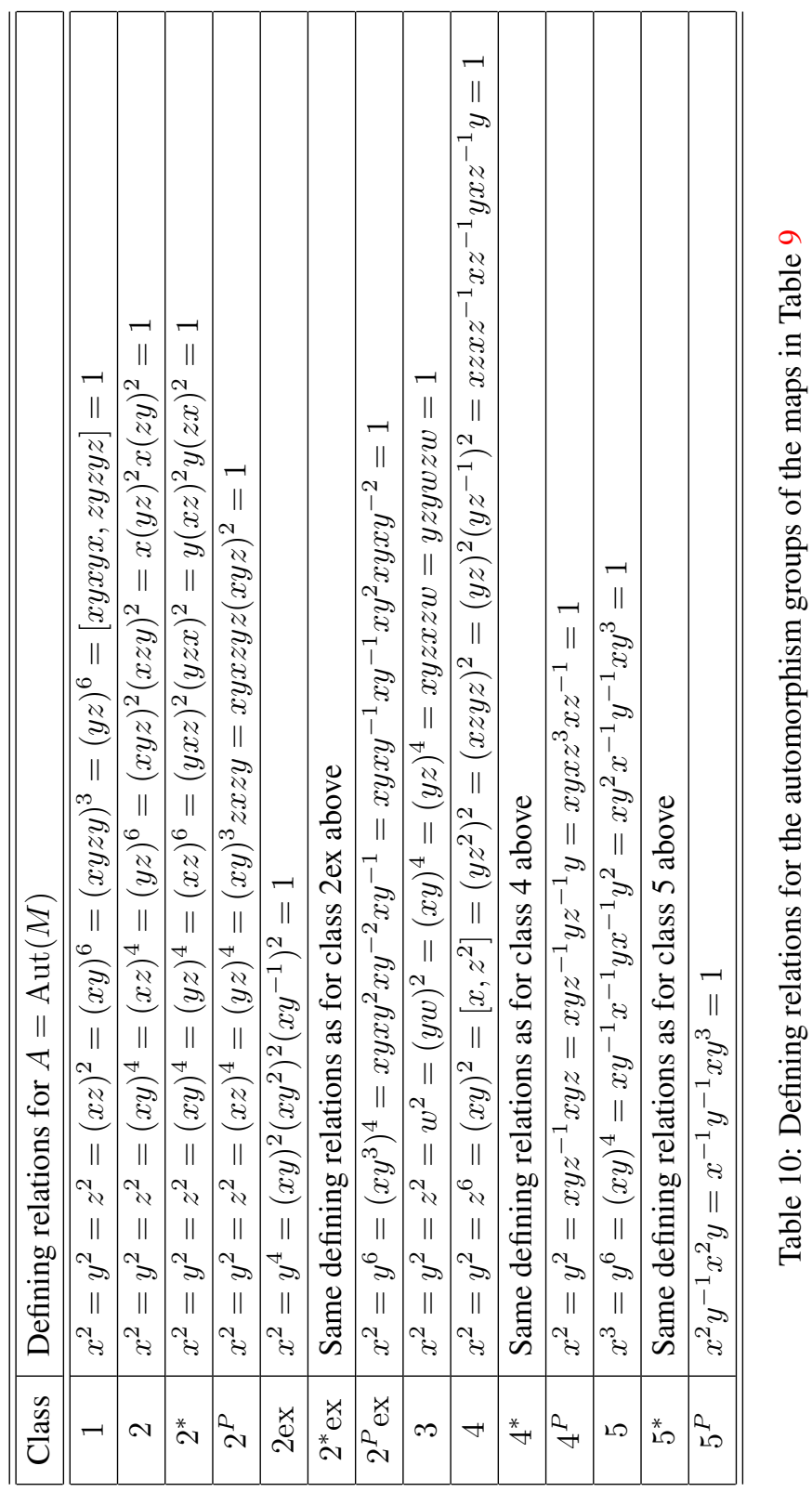




\begin{tabular}{|c|c|c|c|c|c|c|l|}
\hline \hline Class & $|V|$ & $|E|$ & $|F|$ & Genus & $A_{u}, A_{v}$ & $A_{f}, A_{g}$ & Comments \\
\hline \hline 1 & 48 & 144 & 48 & 25 & $D_{6}$ & $D_{6}$ & NSD, Orientable \\
\hline 2 & 120 & 288 & 48 & 61 & $D_{4}, D_{6}$ & $D_{6}$ & Orientable \\
\hline $2^{*}$ & 48 & 288 & 120 & 61 & $D_{6}$ & $D_{4}, D_{6}$ & Map dual to the one above \\
\hline $2^{P}$ & 48 & 288 & 48 & 97 & $D_{6}$ & $D_{6}$ & Non-orientable \\
\hline 2 ex & 48 & 288 & 96 & 73 & $D_{6}$ & $C_{6}$ & Orientable \\
\hline $2^{*}$ ex & 96 & 288 & 48 & 73 & $C_{6}$ & $D_{6}$ & Map dual to the one above \\
\hline $2^{P}$ ex & 96 & 288 & 96 & 49 & $C_{6}$ & $C_{6}$ & SD, Orientable \\
\hline 3 & 240 & 576 & 120 & 109 & $V_{4}, D_{3}$ & $D_{6}, D_{4}$ & Orientable \\
\hline 4 & 240 & 576 & 144 & 97 & $D_{6}, C_{3}$ & $V_{4}$ & Orientable \\
\hline $4^{*}$ & 144 & 576 & 240 & 97 & $V_{4}$ & $D_{6}, C_{3}$ & Map dual to the one above \\
\hline $4^{P}$ & 72 & 576 & 144 & 181 & $D_{4}$ & $V_{4}$ & Orientable \\
\hline 5 & 240 & 576 & 96 & 121 & $C_{4}, C_{6}$ & $C_{6}$ & Orientable \\
\hline $5^{*}$ & 96 & 576 & 240 & 121 & $C_{6}$ & $C_{4}, C_{6}$ & Map dual to the one above \\
\hline $5^{P}$ & 96 & 576 & 96 & 193 & $C_{6}$ & $C_{6}$ & SD, Orientable \\
\hline \hline
\end{tabular}

Table 11: Non-degenerate ET maps of all 14 types with $\operatorname{Aut}(M) \cong \operatorname{Group}(576,8654)$ 


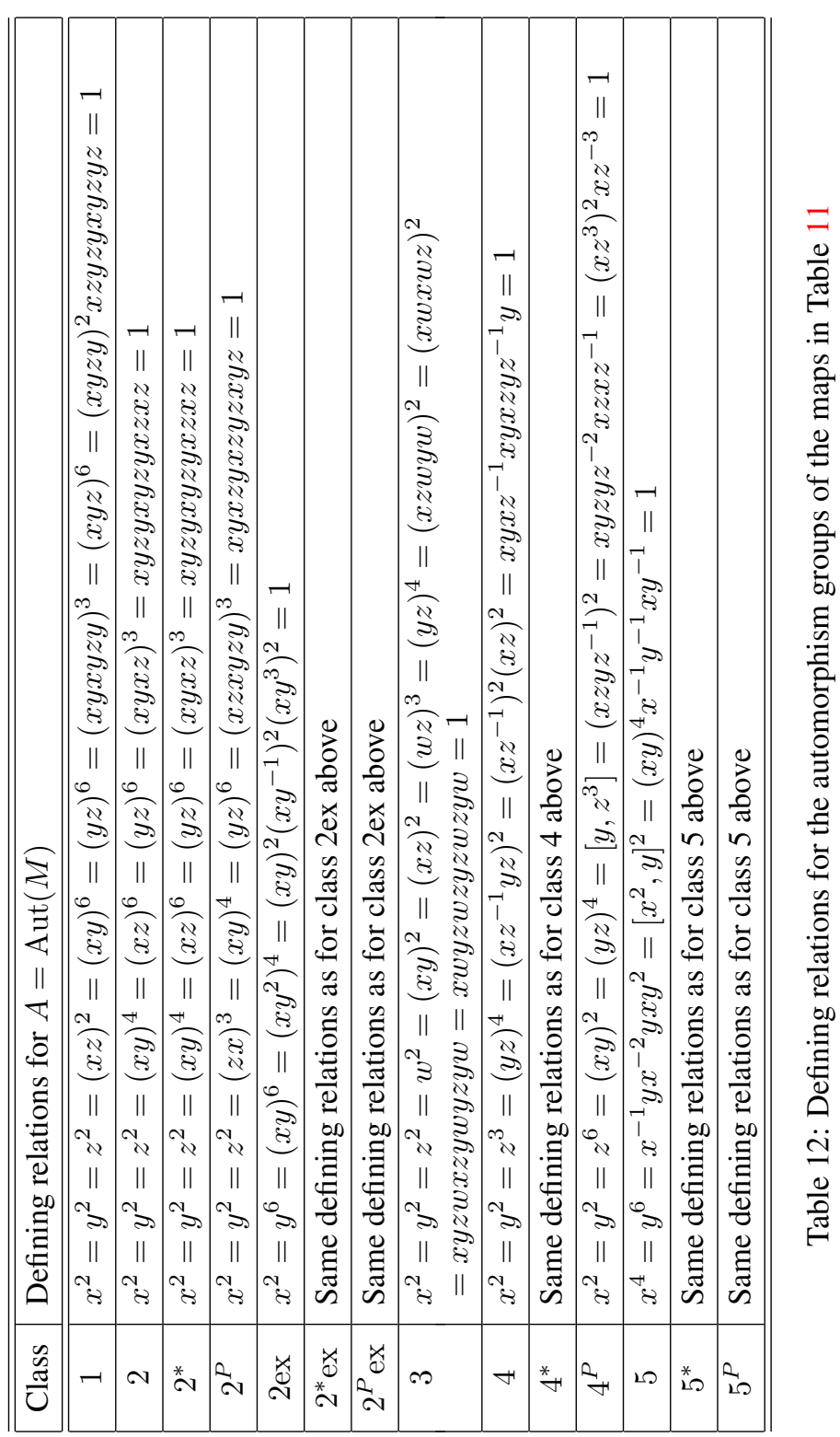

